\title{
High-order exceptional points in supersymmetric arrays
}

\author{
S. M. Zhang $\odot,{ }^{1}$ X. Z. Zhang, ${ }^{2}$ L. Jin $\odot,,^{1, *}$ and Z. Song $\oplus^{1}$ \\ ${ }^{1}$ School of Physics, Nankai University, Tianjin 300071, China \\ ${ }^{2}$ College of Physics and Materials Science, Tianjin Normal University, Tianjin 300387, China
}

(Received 27 September 2019; accepted 18 February 2020; published 16 March 2020)

\begin{abstract}
We employ the intertwining operator technique to synthesize a supersymmetric (SUSY) array of arbitrary size $N$. The synthesized SUSY system is equivalent to a spin $(N-1) / 2$ under an effective magnetic field. By considering an additional imaginary magnetic field, we obtain a generalized parity-time-symmetric nonHermitian Hamiltonian that describes a SUSY array of coupled resonators or waveguides under a gradient gain and loss; all the $N$ energy levels coalesce at an exceptional point (EP), forming an isotropic high-order EP with $N$ states coalescence (EPN). Near the EPN, the scaling exponent of phase rigidity for each eigenstate is $(N-1) / 2$; the eigenfrequency response to the perturbation $\epsilon$ acting on the resonator or waveguide couplings is $\epsilon^{1 / N}$. Our findings reveal the importance of the intertwining operator technique for spectral engineering and exemplify the practical application in non-Hermitian physics.
\end{abstract}

DOI: 10.1103/PhysRevA.101.033820

\section{INTRODUCTION}

The exceptional point (EP) in a non-Hermitian system occurs when eigenstates coalesce [1-3], and is usually associated with the non-Hermitian phase transition [4,5]. In a parity-time $(\mathcal{P} \mathcal{T})$ symmetric non-Hermitian coupled system, the $\mathcal{P} \mathcal{T}$ symmetry of eigenstates spontaneously breaks at the EP [6-16], which determines the exact $\mathcal{P} \mathcal{T}$-symmetric phase and the broken $\mathcal{P} \mathcal{T}$-symmetric phase in this system. In the $\mathcal{P} \mathcal{T}$-symmetric phase, the eigenvalues are real and the intensities oscillate as a result of the nonorthogonality of eigenstates [17]; in the broken $\mathcal{P} \mathcal{T}$-symmetric phase, the intensities exponentially increase because of the complex eigenvalues [7]. Besides the coupled waveguide or resonator lattice, $\mathcal{P} \mathcal{T}$-symmetric systems are simulated by photonic quantum walks [18-20].

The EP has many applications in optics [14,21-26], not limited to nonreciprocal energy transfer [23], unidirectional lasing [27,28], and optical sensing [29,30]. Bidirectional lasing becomes unidirectional lasing when approaching the EP [31]; the direction of lasing is controllable by adjusting the chiral mode of the microresonator. Unidirectional lasing toward a single direction is possible with gain and synthetic magnetic flux [32]. Moreover, the EP is a bifurcation point of the energy levels. Near the EP, the eigenfrequency response to the perturbation exhibits a square-root dependence [29] or a cubic-root [30] dependence. In this regard, EPs are useful for sensing in comparison with the diabolic points; this feature has been verified in optics, cavity optomechanics, cavity

\footnotetext{
*jinliang@nankai.edu.cn

Published by the American Physical Society under the terms of the Creative Commons Attribution 4.0 International license. Further distribution of this work must maintain attribution to the author(s) and the published article's title, journal citation, and DOI.
}

spintronics, and circuit quantum electrodynamics [33-42]. The sensing susceptibility is greatly enhanced near the EPs [43].

Different types of energy level coalescence exist in nonHermitian systems. The most common types of EPs are the two-state coalescence (EP2) that exhibits a square-root dependence on the system parameters $[6-8,29]$ and the threestate coalescence (EP3) that exhibits a cubic-root dependence on the system parameters [30,44-47]. Even four-state coalescence (EP4) is accessible in coupled resonators [48,49]. Recently, a high-order EP of arbitrary order was realized in coupled resonators [50]. And the scaling laws for the eigenvalue and eigenstate confirm the sensitivity of the high-order EP [51-56]; the dynamics near a high-order EP exhibits a power-law dependence on the order of EPs for the maximal amplification [57].

The intertwining operator technique is a useful method for spectral engineering [58,59]. This technique is capable of eliminating a target energy level in the spectrum to create the isospectral SUSY partner [60-63], which has an identical spectrum except for the eliminated target level. In parallel, the intertwining operator technique can add a target energy level or realize a Hamiltonian with spectrum fully constituted by the desirable energy levels [59]. Exactly solvable models with desirable energy levels can be synthesized by employing the intertwining operator technique. Thus, the intertwining operator technique is beneficial for proposing a non-Hermitian Hamiltonian with multiple energy level coalescence. The concept of supersymmetry, originating from quantum field theory [64], has grown in importance in recent years in the research fields of optics and photonics. It is possible to create designed spectra and propose intriguing applications using synthesized system. The SUSY array synthesized through the intertwining operator technique can be utilized for optical sensing [29,30], single mode lasing [60-62], and optical mode converting [63]. An integrated lasing array usually has multiple mode emission. To acquire single mode lasing, one can design 
an isospectral partner SUSY array using the intertwining operator technique. The spectrum of the partner SUSY array is engineered to be constituted by all the excited-state modes except for the ground-state mode of the lasing array. Coupling the partner SUSY array to the lasing array and intentionally inducing loss in the partner SUSY array can enable groundstate single mode lasing [60-62]. The SUSY array can remove the ground-state mode of a multimode light field and manipulate the modal content of the light field through a hierarchical sequence of partner SUSY arrays [63]. The synthetic SUSY array is a hypercube useful for quantum information science [65], and the proposed SUSY array has equally spaced energy levels; thus the SUSY array is capable of realizing a perfect state transfer in which the initial state is exactly mapped from one side of the SUSY array to the other side of it [52].

In this paper, we introduce the intertwining operator technique to propose a non-Hermitian SUSY array of arbitrary size. The energy levels of the proposed SUSY array are equally spaced square-root branches. The non-Hermitian phase transition in the proposed SUSY array is associated with an isotropic high-order EP. In contrast to the anisotropic high-order EP [54], the isotropic high-order EP has identical parameter dependence for independent system parameters in the parameter space. The Hamiltonian of the SUSY array can be understood either as many uncorrelated spin- $1 / 2$ particles in a magnetic field or as a noninteracting bosonic manyparticle system in a two-site model. For an arbitrarily highorder EP, topological properties and the frequency response to perturbation on the resonator couplings are investigated. The isotropic EP in a SUSY array of $N$ sites (EPN) has the phase rigidity scaling exponent $(N-1) / 2$. The eigenfrequency sharply responds to the coupling perturbation $\epsilon$ with the form $\epsilon^{1 / N}$ near the EPN. The results are in accord with topological features of the EPN. The SUSY array is appropriate for perfect state transfer in quantum information science.

The remainder of the paper is organized as follows. In Sec. II, we introduce the intertwining operator technique to synthesize a $\mathcal{P} \mathcal{T}$-symmetric SUSY array. In Sec. III, we investigate the topological properties of the arbitrarily highorder EP through the phase rigidity. In Sec. IV, we focus on the eigenfrequency response to the coupling perturbation to reflect features of the arbitrarily high-order EP. In Sec. V, we summarize the results.

\section{II. $\mathcal{P} \mathcal{T}$-SYMMETRIC SUSY ARRAY}

In this section, we introduce the intertwining operator technique to propose a synthetic SUSY array with all the energy levels equally spaced. The synthetic SUSY is a hypercube, and a hypercube of arbitrary dimension can be synthesized. The schematic of a synthetic six-site SUSY array of coupled resonators (upper panel) or coupled waveguides (lower panel) is shown in Fig. 1(a). The frequency of each resonator (waveguide) is $\omega_{0}$. The resonators (waveguides) are coupled through evanescent tunneling between neighboring ones. The coupling amplitudes of the SUSY array are determined from the intertwining operator technique. In Fig. 1(b), schematics of energy levels of non-Hermitian SUSY arrays with different site numbers are shown; all the levels are equally spaced and (a)

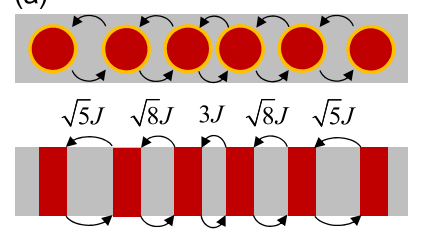

(b)

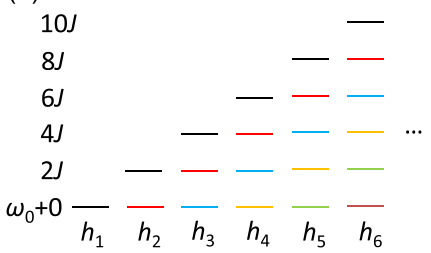

FIG. 1. (a) The SUSY array has a linear gradient on the gain and loss distribution. The resonant frequency is $\omega_{0}$ for each resonator or waveguide. (b) Proposal for engineering the SUSY array.

the energy difference between each pair of neighboring energy levels is $2 J$. To constitute the SUSY array, the couplings are required to be engineered at the proper amplitudes.

In the framework of quantum mechanics, the procedure is as follows. The Hamiltonian $h_{N}$ has $N$ energy levels and we aim to remove an energy level $\varepsilon_{N}$ from $h_{N}$ to construct a target superpartner $h_{N}^{\prime}$. The Hamiltonian $h_{N}$ is factorized into

$$
h_{N}=Q_{N-1} R_{N-1}-\varepsilon_{N} I_{N},
$$

where $Q_{N-1}$ is an $N \times(N-1)$ matrix, $R_{N-1}$ is an $(N-1) \times$ $N$ matrix, and $I_{N}$ is the $N \times N$ identity matrix. The target superpartner Hamiltonian is obtained as

$$
h_{N}^{\prime}=R_{N-1} Q_{N-1}-\varepsilon_{N} I_{N-1} .
$$

$h_{N}^{\prime}$ is an $(N-1) \times(N-1)$ matrix with $(N-1)$ levels identical to $h_{N}$ except for the energy level $\varepsilon_{N}$.

To synthesize the SUSY array, we consider an inverse process and gradually increase the size of the target Hamiltonian by adding target energy levels one by one. Details of synthesizing the SUSY array are displayed as follows. We start with a single-level system $h_{1}$, shift its energy level by $2 J$, and add a zero energy $\left(\varepsilon_{2}=0\right)$ to obtain energy spectrum $\{0,2 J\}$ of the target Hamiltonian $h_{2}$. We factorize the Hamiltonian $h_{1}+2 J=R_{1} Q_{1}$ with $R_{1}=\sqrt{J}(1,1)$ and take $Q_{1}$ as the transpose of $R_{1}$, that is $Q_{1}=R_{1}^{T}$. The factorization is in the form of

$$
h_{1}+2 J=R_{1} Q_{1}=\sqrt{J}\left(\begin{array}{ll}
1 & 1
\end{array}\right) \sqrt{J}\left(\begin{array}{l}
1 \\
1
\end{array}\right)=2 J .
$$

Then, following the intertwining operator technique, we interchange $R_{1}$ and $Q_{1}$ in the matrix product to obtain the target Hamiltonian

$$
h_{2}=Q_{1} R_{1}=\sqrt{J}\left(\begin{array}{l}
1 \\
1
\end{array}\right) \sqrt{J}\left(\begin{array}{ll}
1 & 1
\end{array}\right)=J\left(\begin{array}{ll}
1 & 1 \\
1 & 1
\end{array}\right) .
$$

The target Hamiltonian is a $2 \times 2$ matrix $h_{2}=Q_{1} R_{1}=J \sigma_{x}+$ $J I_{2}$, where $\sigma_{x}$ is the Pauli matrix. Next, we offset the energy by $-J$ to get $h_{2}-J I_{2}=J \sigma_{x}$ and gather the Hermitian SUSY array of $N=2$. The matrix form of $h_{2}$ after subtracting out the term $J_{2}$ is equivalent to the single-particle Hamiltonian for the two-site model. $J \sigma_{x}$ also describes a spin-1/2 particle in an effective magnetic field $J$ along the $x$ direction, that is $\left(B_{x}, B_{y}, B_{z}\right)=(J, 0,0)$.

Furthermore, in order to construct the non-Hermitian Hamiltonian with the EPs, we consider an additional effective imaginary magnetic field $i \gamma$ applied along the $z$ direction; the $\mathcal{P} \mathcal{T}$-symmetric non-Hermitian dimer is obtained with the 
expression $J \sigma_{x}+i \gamma \sigma_{z}$, and the corresponding matrix form is given by

$$
H_{2}=\left(\begin{array}{cc}
\omega_{0}+i \gamma & J \\
J & \omega_{0}-i \gamma
\end{array}\right),
$$

where $\omega_{0}$ is the on-resonator frequency of each resonator or waveguide. $\mathrm{H}_{2}$ has a pair of EP2s at $\gamma= \pm J$, where two eigenstates coalesce to one, $( \pm i, 1)^{T} / \sqrt{2}$.

We repeat the above procedure to construct the nonHermitian SUSY array with high-order EPs. The spectrum of $h_{2}$ is shifted by $2 J$ to $\{2 J, 4 J\}$, and then $\varepsilon_{3}=0$ is added to obtain the spectrum $\{0,2 J, 4 J\}$ of the target Hamiltonian $h_{3}$ [Fig. 1(b)]. We factorize $h_{2}+2 J=R_{2} Q_{2}$ with $R_{2}=Q_{2}^{T}$, $Q_{2}(j, k)=\sqrt{J(j-1)} \delta_{j-1, k}+\sqrt{J(3-j)} \delta_{j, j}$, where $\delta$ means the Kronecker delta function. The factorization gives

$$
h_{2}+2 J I_{2}=R_{2} Q_{2}=J\left(\begin{array}{ll}
3 & 1 \\
1 & 3
\end{array}\right) .
$$

Following the intertwining operator technique, we obtain a $3 \times 3$ Hamiltonian after interchanging $R_{2}$ and $Q_{2}$ in the matrix product, that is

$$
h_{3}=Q_{2} R_{2}=J\left(\begin{array}{ccc}
2 & \sqrt{2} & 0 \\
\sqrt{2} & 2 & \sqrt{2} \\
0 & \sqrt{2} & 2
\end{array}\right) .
$$

The target Hamiltonian $h_{3}$ can be expressed in the form of $h_{3}=Q_{2} R_{2}=J S_{x}+2 J I_{3}$, where $S_{x(z)}$ is the angular momentum operator for spin 1 in the $x(z)$ direction. We remove the overall energy background $2 J I_{3}$ from $h_{3}$ to obtain the Hermitian SUSY array of $N=3 . h_{3}-2 J I_{3}=2 J S_{x}$ describes a spin-1 particle in an effective real magnetic field $(J, 0,0)$.

By considering an effective imaginary field $i \gamma$ applied in the $z$ direction, $(0,0, i \gamma)$, we obtain the $\mathcal{P} \mathcal{T}$-symmetric non-Hermitian trimer $J S_{x}+i \gamma S_{z}$. The corresponding nonHermitian SUSY array is

$$
H_{3}=\left(\begin{array}{ccc}
\omega_{0}+2 i \gamma & \sqrt{2} J & 0 \\
\sqrt{2} J & \omega_{0} & \sqrt{2} J \\
0 & \sqrt{2} J & \omega_{0}-2 i \gamma
\end{array}\right) .
$$

The generalized SUSY array here is the two-particle Hamiltonian for the two-site model in non-Hermitian cases, with the matrix form corresponding to the Hamiltonian described by Eq. (8). The quantum theory of spin (angular momentum) tells us that the energy spectrum of the Hamiltonian $J S_{x}+i \gamma S_{z}$ is restricted to $n \sqrt{J^{2}-\gamma^{2}}$ with $n=-2,0,2$. Each EP of $\mathrm{H}_{3}$ belongs to EP3 with the eigenfrequency $\omega_{0}$ and coalescence eigenstate $(-1, \pm i \sqrt{2}, 1)^{T} / 2$ at the critical condition $\gamma= \pm J$.

To synthesize the $4 \times 4$ SUSY array, we shift all the energy levels of $h_{3}$ by $2 J$ to obtain $\{2 J, 4 J, 6 J\}$ and add a zero level to compose the spectrum $\{0,2 J, 4 J, 6 J\}$ of the target Hamiltonian $h_{4}$. We factorize $h_{3}$ in the form of

$$
h_{3}+2 J I_{3}=R_{3} Q_{3}=J\left(\begin{array}{ccc}
4 & \sqrt{2} & 0 \\
\sqrt{2} & 4 & \sqrt{2} \\
0 & \sqrt{2} & 4
\end{array}\right) .
$$

Following the intertwining operator technique, we obtain the $4 \times 4$ target Hamiltonian

$$
h_{4}=Q_{3} R_{3}=J\left(\begin{array}{cccc}
3 & \sqrt{3} & 0 & 0 \\
\sqrt{3} & 3 & 2 & 0 \\
0 & 2 & 3 & \sqrt{3} \\
0 & 0 & \sqrt{3} & 3
\end{array}\right) .
$$

We offset energy $3 J$ from $h_{4}$ to obtain the Hermitian SUSY array of $N=4$, which describes a spin-3/2 particle in an effective real magnetic field $(J, 0,0)$. The non-Hermitian generalization gives $J S_{x}+i \gamma S_{z}$, where $S_{x(z)}$ is the angular momentum operator for spin $3 / 2$ in the $x(z)$ direction. The matrix form of the SUSY array is

$$
H_{4}=\left(\begin{array}{cccc}
\omega_{0}+3 i \gamma & \sqrt{3} J & 0 & 0 \\
\sqrt{3} J & \omega_{0}+i \gamma & 2 J & 0 \\
0 & 2 J & \omega_{0}-i \gamma & \sqrt{3} J \\
0 & 0 & \sqrt{3} J & \omega_{0}-3 i \gamma
\end{array}\right) .
$$

The $J$ related terms are the couplings between neighbor resonators induced by the evanescent fields, and depend on the distance between them. The distribution of gain and loss in the SUSY array has a gradient. The $\mathcal{P} \mathcal{T}$-symmetric nonHermitian $4 \times 4$ SUSY array is the three-particle Hamiltonian for the two-site model, the spectrum of which is restricted to $n \sqrt{J^{2}-\gamma^{2}}$ with $n=-3,-1,1,3$, with the appearance of EP4 at $\gamma= \pm J$.

We can synthesize the SUSY array of arbitrary size via repeating the same procedure. The $\mathcal{P} \mathcal{T}$-symmetric nonHermitian $5 \times 5$ SUSY array has the form

$$
H_{5}=\omega_{0} I_{5}+\left(\begin{array}{ccccc}
4 i \gamma & 2 J & 0 & 0 & 0 \\
2 J & 2 i \gamma & \sqrt{6} J & 0 & 0 \\
0 & \sqrt{6} J & 0 & \sqrt{6} J & 0 \\
0 & 0 & \sqrt{6} J & -2 i \gamma & 2 J \\
0 & 0 & 0 & 2 J & -4 i \gamma
\end{array}\right) .
$$

The $\mathcal{P} \mathcal{T}$-symmetric non-Hermitian $6 \times 6$ SUSY array illustrated in Fig. 1(a) has the form

$$
H_{6}=\omega_{0} I_{6}+\left(\begin{array}{cccccc}
5 i \gamma & \sqrt{5} J & 0 & 0 & 0 & 0 \\
\sqrt{5} J & 3 i \gamma & \sqrt{8} J & 0 & 0 & 0 \\
0 & \sqrt{8} J & i \gamma & 3 J & 0 & 0 \\
0 & 0 & 3 J & -i \gamma & \sqrt{8} J & 0 \\
0 & 0 & 0 & \sqrt{8} J & -3 i \gamma & \sqrt{5} J \\
0 & 0 & 0 & 0 & \sqrt{5} J & -5 i \gamma
\end{array}\right) .
$$

The SUSY arrays $H_{5}$ and $H_{6}$ are the four-particle and fiveparticle Hamiltonians for the two-site model [56], respectively. In both cases, the EP occurs at $\gamma= \pm J$, but is EP5 in $H_{5}$ and EP6 in $H_{6}$. Recently, experimental investigation of high-order EPs has sprung up rapidly. For example, a fifthorder EP can be designed via tuning parameters in nitrogenvacancy centers [66]; a $\mathcal{P} \mathcal{T}$-symmetric electronic circuit has been proposed to study sensing at a sixth-order EP [67].

In general, we shift all the energy levels of $h_{N-1}$ by $2 J$ to obtain a spectrum $\{2 J, 4 J, \ldots, 2(N-1) J\}$ and add $\varepsilon_{N}=0$ by the intertwining operator technique to 
obtain $h_{N}$ with the spectrum $\{0,2 J, \ldots, 2(N-1) J\}$. We obtain $h_{N}=Q_{N-1} R_{N-1}=J S_{x}+(N-1) J I_{N}$ with $R_{N-1}=$ $Q_{N-1}^{T}, Q_{N-1}(j, k)=\sqrt{J(j-1)} \delta_{j-1, k}+\sqrt{J(N-j)} \delta_{j, j}$, and $S_{x(z)}$ is the angular momentum operator for spin $(N-1) / 2$ in the $x(z)$ direction. Removing the offset energy $(N-1) J$ and introducing an imaginary magnetic field in the $z$ direction, we obtain the $\mathcal{P} \mathcal{T}$-symmetric non-Hermitian $N \times N$ model $J S_{x}+i \gamma S_{z}$ [52]. $H_{N}$ describes an $N$-site $\mathcal{P} \mathcal{T}$-symmetric nonHermitian SUSY array [61-63]. The imaginary magnetic field corresponds to tilted on-site imaginary potentials in the form of gain and loss, which linearly depend on the site number. The concise form of $H_{N}$ is given by

$$
\begin{aligned}
H_{N}= & \sum_{m=1}^{N-1} J \sqrt{m(N-m)}(|m\rangle\langle m+1|+\text { H.c. }) \\
& +\sum_{m=1}^{N}\left[\omega_{0}+i \gamma(N+1-2 m)\right]|m\rangle\langle m| .
\end{aligned}
$$

The non-Hermitian generalized SUSY array is synthesized by a recursive bosonic quantization technique in coupled resonators or waveguides [53].

The first line in $H_{N}$ is the Hermitian SUSY array [65]. The energy of $J S_{x}$ relates to the (quantized) possible value of spin angular momentum in the $x$ direction, which is $n J$ for the integer $n=-(N-1),-(N-3), \ldots,(N-3),(N-1) . H_{N}$ describes a spin- $(N-1) / 2$ particle in an effective magnetic field $\left(B_{x}, B_{y}, B_{z}\right)=(J, 0, i \gamma)$. This indicates that the SUSY array $H_{N}$ has the frequency

$$
\omega_{N, n}=\omega_{0}+n \sqrt{J^{2}-\gamma^{2}} .
$$

Notably, the EPs of $H_{N}$ are exactly EPNs at $\gamma= \pm J$, where all the levels are square-root branches and coalesce to the resonant frequency $\omega_{0}$.

Furthermore, introducing the angular momentum operators $S_{x}=a_{1}^{\dagger} a_{2}+a_{2}^{\dagger} a_{1}, S_{y}=i a_{2}^{\dagger} a_{1}-i a_{1}^{\dagger} a_{2}$, and $S_{z}=a_{1}^{\dagger} a_{1}-a_{2}^{\dagger} a_{2}$, the Hamiltonian $J S_{x}+i \gamma S_{z}=J\left(a_{1}^{\dagger} a_{2}+a_{2}^{\dagger} a_{1}\right)+i \gamma\left(a_{1}^{\dagger} a_{1}-\right.$ $\left.a_{2}^{\dagger} a_{2}\right) \equiv H_{\text {two-site }}$ can be alternatively understood as the noninteracting bosonic many-particle system in a $\mathcal{P} \mathcal{T}$-symmetric non-Hermitian two-site model [56], where $a_{1(2)}^{\dagger}$ and $a_{1(2)}$ are the creation and annihilation operators of the first (second) site, respectively. $\left[S_{a}, S_{b}\right]=2 i \epsilon_{a b c} S_{c}$, where $\epsilon_{a b c}$ is the LeviCivita symbol and $a, b, c$ are $x, y, z$. The commutation relation is equivalent to that of Pauli matrices with spin $1 / 2$.

The basis set for the single-particle system is chosen as $|1\rangle_{1}=a_{1}^{\dagger}|\mathrm{vac}\rangle,|2\rangle_{1}=a_{2}^{\dagger}|\mathrm{vac}\rangle . H_{2}$ in Eq. (5) is $H_{\text {two-site }}$ in the single-particle basis. If we consider the two-particle problem, the basis set is $|1\rangle_{2}=\left(a_{1}^{\dagger}\right)^{2} / \sqrt{2}|\mathrm{vac}\rangle$, $|2\rangle_{2}=a_{1}^{\dagger} a_{2}^{\dagger}|\mathrm{vac}\rangle,|3\rangle_{2}=\left(a_{2}^{\dagger}\right)^{2} / \sqrt{2} \mid$ vac $\rangle$. The factor $1 / \sqrt{2}$ in the basis is not only the normalization factor to ensure ${ }_{2}\langle m \mid n\rangle_{2}=\delta_{m n}(m, n=1,2,3)$, but also the normalization factor in the Fock representation for the occupation number of $2 . H_{3}$ in Eq. (8) is $H_{\text {two-site }}$ in the twoparticle basis. Moreover, the basis set for three particles is $|1\rangle_{3}=\left(a_{1}^{\dagger}\right)^{3} / \sqrt{6}|\mathrm{vac}\rangle,|2\rangle_{3}=\left(a_{1}^{\dagger}\right)^{2} a_{2}^{\dagger} / \sqrt{2}|\mathrm{vac}\rangle,|3\rangle_{3}=$ $a_{1}^{\dagger}\left(a_{2}^{\dagger}\right)^{2} / \sqrt{2}|\mathrm{vac}\rangle,|4\rangle_{3}=\left(a_{2}^{\dagger}\right)^{3} / \sqrt{6}|\mathrm{vac}\rangle . H_{4}$ in Eq. (11) is $H_{\text {two-site }}$ in the three-particle basis. $H_{2}$ [29], $H_{3}$ [30], and $H_{4}$ [51] are experimentally realized in different physical setups. In general, the basis for an $(N-1)$-particle system is $|l\rangle_{N-1}=\left(a_{1}^{\dagger}\right)^{N-1-l}\left(a_{2}^{\dagger}\right)^{l} / \sqrt{(N-1-l) ! l !} \mid$ vac $\rangle$, where $l=$ $0,1, \ldots, N-1$ and the subscript in the basis stands for the number of particles. $H_{\text {two-site }}$ in the basis $\left\{|l\rangle_{N-1}\right\}$ gives $H_{N}$ in Eq. (14).

The notion of SUSY plays an important role for plenty of intriguing optical properties and functionalities as well as for a number of practical applications of optical metamaterials [60-63]. The non-Hermitian SUSY array also provides a promising platform for the study of the topology of arbitrary high-order EPs. The geometric topological properties reflect the order of EPs; they are the essential features of different EPs. The topological features of different EPs are also captured by the phase rigidity scaling exponents [49].

\section{PHASE RIGIDITY}

The phase rigidity is defined as

$$
r=\left\langle\psi^{*} \mid \psi\right\rangle /\langle\psi \mid \psi\rangle,
$$

and $r$ reflects the mixing of different states $\psi^{*}$ and $\psi[68,69]$. We consider the phase rigidities associated with the eigenstates of the SUSY array. At the EPs $\gamma_{\mathrm{EP}}= \pm J$, the coalescence state is $\left|u_{1}\right\rangle=\left|u_{2}\right\rangle=( \pm i, 1)^{T} / \sqrt{2}=\left|u_{\mathrm{EP}}\right\rangle$ for the nonHermitian Hamiltonian $H_{2}$. The eigenfrequencies are $\omega_{0}+$ $\varepsilon_{1}$ and $\omega_{0}+\varepsilon_{2}$, where $\varepsilon_{1}=\sqrt{J^{2}-\gamma^{2}}, \varepsilon_{2}=-\sqrt{J^{2}-\gamma^{2}}$. The eigenstates satisfy $H_{2}\left|u_{j}\right\rangle=\left(\omega_{0}+\varepsilon_{j}\right)\left|u_{j}\right\rangle \quad(j=1,2)$ with $\left|u_{1}\right\rangle=\left(e^{i \theta}, 1\right)^{T} / \sqrt{2}$ and $\left|u_{2}\right\rangle=\left(-e^{-i \theta}, 1\right)^{T} / \sqrt{2}$, where $\cos \theta=\sqrt{J^{2}-\gamma^{2}} / J$ and $\sin \theta=\gamma / J . H_{N}$ at the EPN has only one coalescence state for arbitrary $N$. Moreover, the eigenstates of $H_{N}$ can be expressed as the direct product of $\left|u_{1}\right\rangle$ and $\left|u_{2}\right\rangle$. Straightforward calculation indicates $r_{\mathrm{EP}}=0$ for $H_{N}$ because of $\left\langle u_{\mathrm{EP}}^{*} \mid u_{\mathrm{EP}}\right\rangle=0$. The phase rigidity has a scaling behavior near the EPs, $|r|=\left|\gamma-\gamma_{\mathrm{EP}}\right|^{v}$, where $v$ is the scaling exponent and describes the topological feature of EPs.

The basis and eigenstates of the $(N-1)$-particle Hamiltonian are chosen under the Fock representation. Considering the direct product representation that employs the singleparticle eigenstates as the basis, the expression of eigenstates of the $(N-1)$-particle system is the direct product of the $N-1$ numbers of single-particle eigenstates $\left|u_{1}\right\rangle$ and $\left|u_{2}\right\rangle$. The general expression of the normalized eigenstate of a spin- $(N-1) / 2$ system in the direct product representation is $\left(\left|u_{1}\right\rangle\right)^{l} \otimes\left(\left|u_{2}\right\rangle\right)^{N-1-l}$ with the eigenvalue $l \varepsilon_{1}+$ $(N-1-l) \varepsilon_{2}$, where the integer $l \in[0, N-1]$. Although the expressions of Hamiltonians and eigenstates are formally different under the two representations, the topological properties of EPs remain unchanged. When approaching the EPN, the ratio of phase rigidities of the eigenstates under the Fock representation to that under the direct product representation is a constant, which does not affect the scaling law near the EPN. The phase rigidity $r$ near the EPN under direct product representation is $r=r_{1}^{l} r_{2}^{N-1-l}$; the scaling exponent $v$ for EPN is $v=\log _{10}\left|r_{1}^{l} r_{2}^{N-1-l}\right| / \log _{10}\left|\gamma-\gamma_{\mathrm{EP}}\right|=$ $(N-1) / 2$ (see Appendix A). The analysis of the scaling exponents of higher-order EPs is numerically verified in Fig. 2, where the phase rigidities and scaling exponents of EP3, EP4, EP5, and EP6 under the Fock representation Hamiltonian [Eq. (14)] are shown for $N=3,4,5,6$. The scaling exponents 
(a)

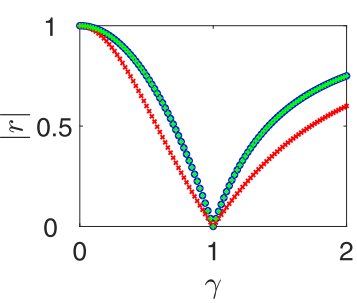

(c)

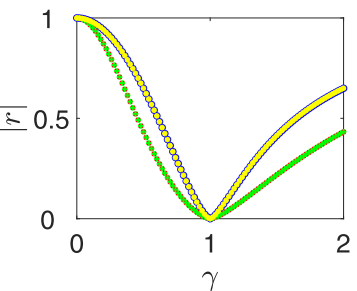

(e)

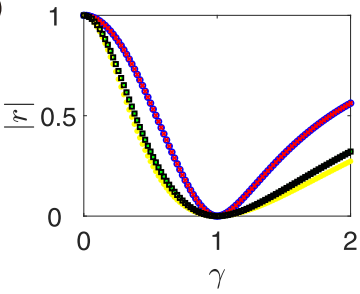

(g)

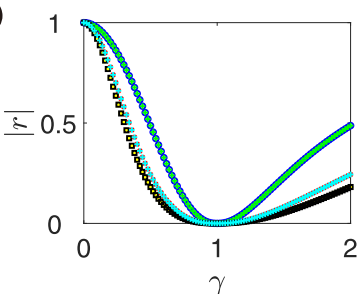

(b)

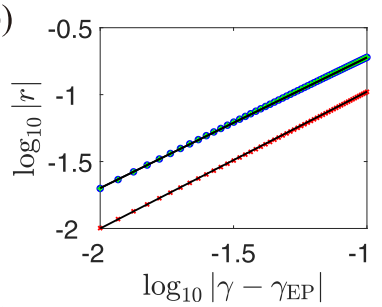

(d)

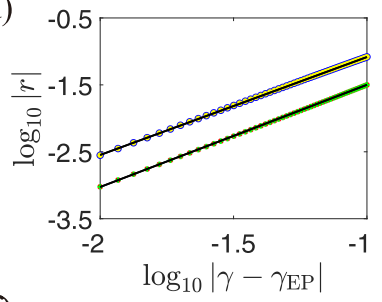

(f)

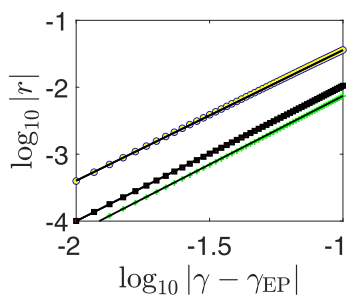

(h)

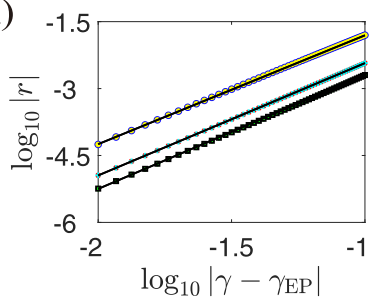

FIG. 2. Phase rigidities and scaling exponents of the high-order EP. (a), (c), (e), (g) and (b), (d), (f), (h) are the numerical results of absolute values of the phase rigidities $|r|=\left|\left\langle\psi^{*} \mid \psi\right\rangle\right| /\langle\psi \mid \psi\rangle$ and the logarithmic relationship between $|r|$ and $\left|\gamma-\gamma_{\mathrm{EP}}\right|$ of energy levels at EP3, EP4, EP5 and EP6, respectively. The phase rigidities are depicted in the left panel; the scaling exponents in the right panel are $1,3 / 2,2$, and 5/2 associated with EP3, EP4, EP5, and EP6. The black lines are the linear fits. The system parameter is $J=1$ and all the EPs are at $\gamma_{\mathrm{EP}}=1$.

are 1.0, 1.5, 2.0 and 2.5 for the EP3, EP4, EP5, and EP6, respectively. Notably, there exists a zero-energy flat band for odd $N$, which participates in the coalescence of eigenstates (see Appendix B). Thus it exhibits a scaling behavior identical to that of other levels. We emphasis that the high-order EPs in the SUSY array are isotropic. Replacing $i \gamma$ by $\Delta+i \gamma$, we observe the scaling exponent $v=(N-1) / 2$ for tuning the detuning $\Delta$ to approach the EPNs $|r|=\left|\Delta-\Delta_{\mathrm{EP}}\right|^{\nu}$. The scaling exponent of the phase rigidity is robust to the perturbation $[54,70]$. For an anisotropic EPN in the system with asymmetric couplings, the scaling exponents of the phase rigidity are $(N-1) / 2$ and $N-1$ when approaching EPN from two independent parameters, respectively [54].

\section{EIGENFREQUENCY RESPONSE TO PERTURBATION}

The non-Hermitian SUSY array at the high-order EP enhances the susceptibility in optical sensing, and the frequency response near the high-order EP is greatly increased

$[29,30,43,70]$. Near the high-order EP in the non-Hermitian SUSY array, a remarkable point is the enhanced frequency response to the detuning as well as the coupling when the array is subjected to the perturbation $\epsilon$. The SUSY array is a hypercube with high symmetry, the response to $\epsilon$ acting on the coupling appears to have a response similar to that acting on the detuning when approaching an EPN. And the eigenfrequency response is $\sim \epsilon^{1 / 2}$ for EP2 $[29,38]$ and $\sim \epsilon^{1 / 3}$ for EP3 [30], which are distinct from the linear response to the perturbation strength $\sim \epsilon$ near the degeneracy point in Hermitian systems. The sharp response is a typical feature of the EP that paves the way for the application of sensors. Moreover, the SUSY array we constructed holds an arbitrary $\operatorname{EPN}(N \geqslant 2)$ at $\gamma= \pm J$, which has striking features.

According to the Newton-Puiseux series expansion [71,72], the frequency splitting $\omega_{l}$ is a function of the perturbation $c_{1} \epsilon^{1 / N}+c_{2} \epsilon^{2 / N}+\cdots$ [70], where the corresponding coefficients $c_{1}, c_{2}, \ldots$ are complex numbers. In the perturbation theory, the unperturbed Hamiltonian is $H_{N}$, and the perturbation Hamiltonian is $H^{\prime}\left(H^{\prime} \ll H_{N}\right)$. The SUSY array under perturbations reads $H_{N \times N}=H_{N}+H^{\prime}$. The eigenfrequency of $H_{N \times N}$ and the expansion coefficients $c_{1}$ and $c_{2}$ are determined by the equation $\operatorname{det}\left[H_{N \times N}-\omega_{l} I_{N}\right]=0$ through substituting the first two terms $c_{1} \epsilon^{1 / N}+c_{2} \epsilon^{2 / N}$ of $\omega_{l}$. Because $\epsilon \ll J$, the higher order terms of $\epsilon$ can be neglected. Notably, in general cases, $\omega_{l} \approx c_{1} \epsilon^{1 / N}$ for comparable $c_{1} \sim c_{2}$. We also show that the eigenfrequency response to the coupling perturbation characterized by the order of magnitude $\sim \epsilon^{1 / N}$ near an EPN in the SUSY array is similar to that of the detuning perturbation.

The coupling is determined by the distance between resonators or waveguides. In practice, the resonator frequency can be accurately fabricated, and the coupling $J$ may have imperfections. Thus, it is reasonable to consider the resonator coupling perturbations for the EPN of the SUSY array $H_{N}$. We take the example in Fig. 3 that all the coupling terms have perturbations, that is, the $J$ related terms are $J \sqrt{m(N-m)}+$ $\epsilon(m=1,2, \ldots N-1)$.

Figure 3 depicts the energy levels, frequency splitting, as well as the logarithmic plots of the frequency splitting as a function of coupling perturbation $\epsilon$. The coupling perturbation $\epsilon$ presents in each coupling $J \sqrt{m(N-m)}$. The whole spectrum is symmetric about zero energy due to the equal amount of $\epsilon$ chosen without breaking the chiral symmetry of $H_{N}$. The frequency splitting response proportional to $\epsilon^{1 / N}$ is shown in Fig. 3; this differs from the response to the coupling perturbation $J+\epsilon$ instead of $J$ in Eq. (15), which leads to the square-root dependence $\sim \epsilon^{1 / 2}$. The frequency splitting dependence on the coupling perturbation between the first two cavities exhibits slopes of $1 / 3$ and $1 / 4$ for $H_{3}$ and $H_{4}$ in the logarithmic plots; this reveals the cubic-root and quarticroot dependences near the EP3 and EP4 (see Appendix C). In general cases, the coupling perturbation $\epsilon$ in an arbitrary resonator of the SUSY array leads to the similar response $\omega_{l} \approx c_{1} \epsilon^{1 / N}$; besides, we can observe the response is in the order of $\omega_{l} \approx c_{2} \epsilon^{2 / N}$. The frequency splitting response as a function of coupling perturbation is identical to the detuning perturbation $[55,56]$, which reflects the high symmetry feature of the hypercube (SUSY array). 
(a)

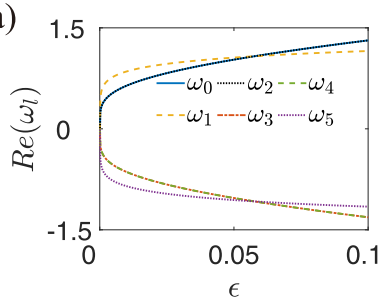

(c)

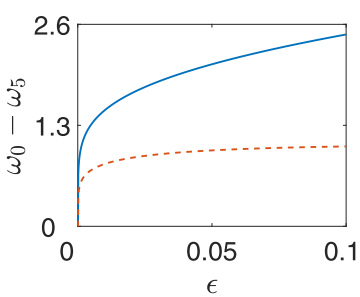

(e)

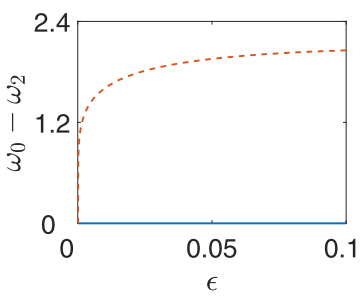

(b)

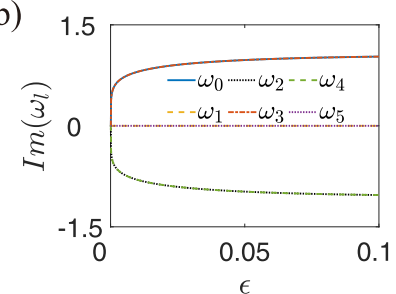

(d)

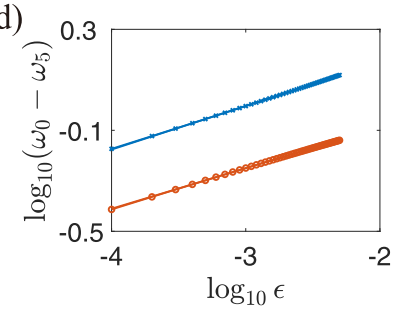

(f)

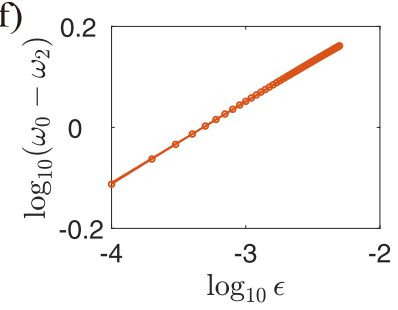

FIG. 3. Energy levels and frequency splittings near the EP6. (a) Real and (b) imaginary parts of eigenvalues $\omega_{l}, l=$ $0,1,2,3,4,5$. (c) and (e) are the frequency splittings between two bands $\omega_{0}, \omega_{5}$ and $\omega_{0}, \omega_{2}$. (d) and (f) are the logarithmic scales of (c) and (e), respectively. In (c), (e) and (d), (f) dashed orange (solid cyan) lines and orange circles (cyan crosses) correspond to the imaginary (real) parts. The slopes are $1 / 6$ in (d) and (f). The system parameters are $\gamma=J=1$.

\section{CONCLUSION}

The intertwining operator technique is an important approach for spectral engineering. We employ the intertwining operator technique to propose a non-Hermitian SUSY array with arbitrarily high-order exceptional points. The Hamiltonian of the proposed array is a non-Hermitian generalized SUSY lattice chain for perfect state transfer in quantum information science, which is equivalent to a noninteracting many-particle Hamiltonian of the two-site non-Hermitian $\mathcal{P T}$-symmetric dimer. At the EPN of the SUSY array with $N$ coupled resonators or waveguides, all the energy levels are equally spaced, being square-root branches and coalescing at the EPN. The phase rigidity of each eigenstate reaches zero and the scaling exponent is $(N-1) / 2$ for the EPN; the eigenfrequency response to perturbation $\epsilon$ is $\epsilon^{1 / N}$ for coupling amplitude perturbation in certain resonators or waveguides of the SUSY array. The intertwining operator technique provides a promising method for synthesizing artificial optical metamaterials.

\section{ACKNOWLEDGMENT}

We acknowledge the support of the National Natural Science Foundation of China (Grants No. 11975128, No. 11975166, and No. 11874225).

\section{APPENDIX A: PHASE RIGIDITY}

The eigenvalues of $H_{2}$ are frequencies $\omega_{0}+\varepsilon_{1}$ and $\omega_{0}+$ $\varepsilon_{2}$, where $\varepsilon_{1}=\sqrt{J^{2}-\gamma^{2}}, \varepsilon_{2}=-\sqrt{J^{2}-\gamma^{2}}$. The eigenstates satisfy $H_{2}\left|u_{j}\right\rangle=\left(\omega_{0}+\varepsilon_{j}\right)\left|u_{j}\right\rangle$ with the expressions $\left|u_{1}\right\rangle=\left(e^{i \theta}, 1\right)^{T} / \sqrt{2},\left|u_{2}\right\rangle=\left(-e^{-i \theta}, 1\right)^{T} / \sqrt{2}$, where $\cos \theta=$ $\sqrt{J^{2}-\gamma^{2}} / J$ and $\sin \theta=\gamma / J$. We start with the eigenstate $\left|u_{1}\right\rangle$ of the $2 \times 2$ Hamiltonian $H_{2}$, where $J$ and $\gamma$ are positive real numbers without loss of generality. At the EP2 $\gamma_{\mathrm{EP}}=J$, $\left|u_{1}\right\rangle=(i, 1)^{T} / \sqrt{2}$, the phase rigidity vanishes with $r_{\mathrm{EP}}=0$.

For $J \geqslant \gamma$, the phase rigidity $r_{1}$ is

$$
r_{1}=\left|\left\langle u_{1}^{*} \mid u_{1}\right\rangle /\left\langle u_{1} \mid u_{1}\right\rangle\right|=\sqrt{1-\gamma^{2} / J^{2}} .
$$

The corresponding scaling exponent $v$ can be expressed as

$$
v=\frac{\log _{10}|r|}{\log _{10}\left|\gamma-\gamma_{\mathrm{EP}}\right|}=\frac{1}{2} \lim _{\gamma \rightarrow J} \frac{\log _{10}\left(\frac{J-\gamma}{J} \frac{J+\gamma}{J}\right)}{\log _{10}(J-\gamma)}=\frac{1}{2} .
$$

For $\gamma \geqslant J$, the phase rigidity $r_{1}$ is

$$
r_{1}=\left|\left\langle u_{1}^{*} \mid u_{1}\right\rangle /\left\langle u_{1} \mid u_{1}\right\rangle\right|=\sqrt{1-J^{2} / \gamma^{2}} .
$$

The corresponding scaling exponent $v$ can be expressed as

$$
v=\frac{\log _{10}|r|}{\log _{10}\left|\gamma-\gamma_{\mathrm{EP}}\right|}=\frac{1}{2} \lim _{\gamma \rightarrow J} \frac{\log _{10}\left(\frac{\gamma-J}{\gamma} \frac{\gamma+J}{\gamma}\right)}{\log _{10}(\gamma-J)}=\frac{1}{2} .
$$

For the other eigenstate $\left|u_{2}\right\rangle$, we have the same conclusion that $r_{2}=\sqrt{1-\gamma^{2} / J^{2}}$ for $J \geqslant \gamma$ and $r_{2}=\sqrt{1-J^{2} / \gamma^{2}}$ for $\gamma \geqslant$ $J$. Therefore, the scaling exponents are both $1 / 2$ for the two eigenstates.

In general, the eigenstate for the $N \times N$ Hamiltonian $H_{N}$ with eigenvalue $l \varepsilon_{1}+(N-1-l) \varepsilon_{2}$ is given by $\left(\left|u_{1}\right\rangle\right)^{l} \otimes$ $\left(\left|u_{2}\right\rangle\right)^{N-1-l}$, where $l=0,1, \ldots, N-1$. Therefore, the phase rigidity vanishes with $r_{\mathrm{EP}}=0$ at $\mathrm{EPN} \gamma_{\mathrm{EP}}=J$ and the corresponding phase rigidity $r$ is

$$
\begin{aligned}
r & =\left|\frac{\left\langle\left(u_{1}^{*}\right)^{l}\left(u_{2}^{*}\right)^{N-1-l} \mid u_{1}^{l} u_{2}^{N-1-l}\right\rangle}{\left\langle u_{1}^{l} u_{2}^{N-1-l} \mid u_{1}^{l} u_{2}^{N-1-l}\right\rangle}\right| \\
& =\left|\frac{\left\langle\left(u_{1}^{*}\right)^{l} \mid u_{1}^{l}\right\rangle\left\langle\left(u_{2}^{*}\right)^{N-1-l} \mid u_{2}^{N-1-l}\right\rangle}{\left\langle u_{1}^{l} \mid u_{1}^{l}\right\rangle\left\langle u_{2}^{N-1-l} \mid u_{2}^{N-1-l}\right\rangle}\right| \\
& =r_{1}^{l} r_{2}^{N-1-l} .
\end{aligned}
$$

The scaling exponent $v$ for EPN is

$$
\begin{aligned}
v & =\frac{\log _{10}\left|r_{1}^{l} r_{2}^{N-1-l}\right|}{\log _{10}\left|\gamma-\gamma_{\mathrm{EP}}\right|} \\
& =\frac{\log _{10}\left|r_{1}\right|^{l}}{\log _{10}\left|\gamma-\gamma_{\mathrm{EP}}\right|}+\frac{\log _{10}\left|r_{2}\right|^{N-1-l}}{\log _{10}\left|\gamma-\gamma_{\mathrm{EP}}\right|} \\
& =\frac{N-1}{2} .
\end{aligned}
$$

\section{APPENDIX B: ENERGY BANDS FOR ODD $N$}

A zero-energy flat band exists in $H_{N}$ when $N$ is odd because $H_{N}$ has chiral symmetry; therefore, the spectrum of $H_{N}$ is symmetric about the zero energy, and there is a zeroenergy flat band if the system has an odd number of energy 
(a)

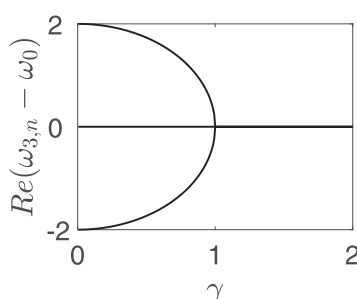

(c)

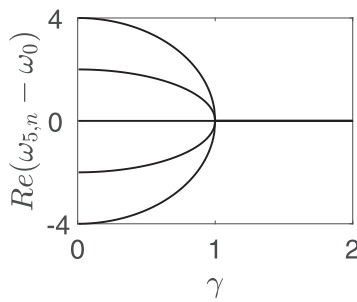

(b)

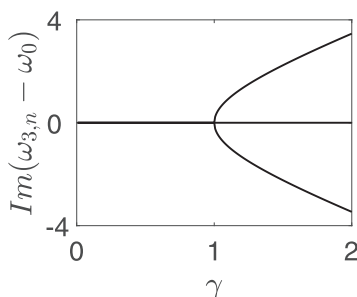

(d)

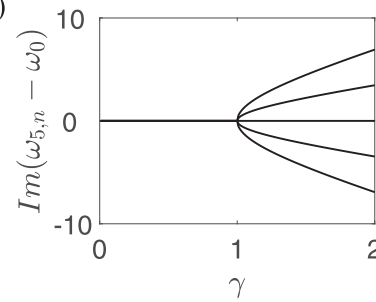

FIG. 4. Energy levels [Eq. (15)] of the SUSY array including a zero-energy level. (a) Real and (b) imaginary parts of $H_{3}$. (c) Real and (d) imaginary parts of $H_{5}$. The system parameter is $J=1$.

levels. At the EP $\gamma= \pm J$, all energy levels coalesce, $H_{N}$ is nondiagonalizable and reduces to a Jordan block,

$$
\mathbf{D}_{N}=\left(d_{m n}\right)= \begin{cases}\omega_{0}, & m=n, \\ 1, & m=n-1, \\ 0, & \text { otherwise. }\end{cases}
$$

Figure 4 shows the spectra of $H_{3}$ [Eq. (8)] and $H_{5}$ [Eq. (12)] as a function of the non-Hermiticity $\gamma$. Both $H_{3}$ and $H_{5}$ hold a zero-energy band, which participates in the coalescence at the $\mathrm{EP} J=\gamma$.

(a)

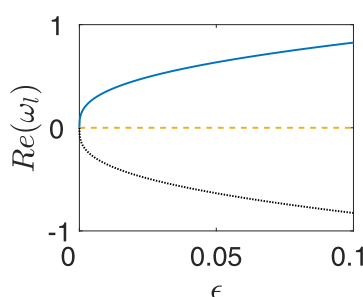

(c)

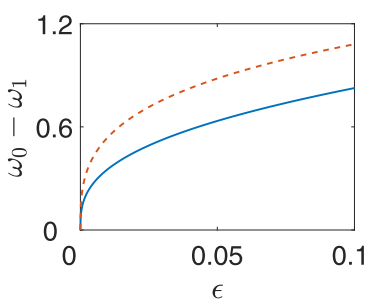

(b)

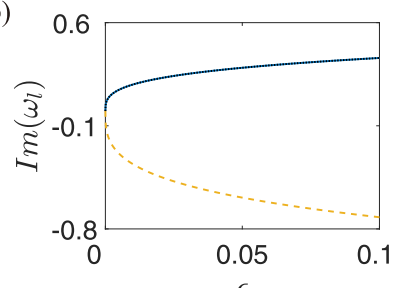

(d)

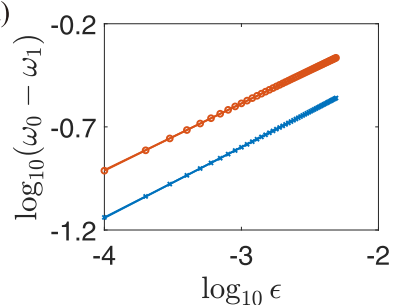

FIG. 5. Energy levels and frequency splittings near the EP3. (a) Real and (b) imaginary parts of three eigenenergies with different colors and line styles. (c) Frequency splitting between $\omega_{0}$ and $\omega_{1}$. (d) Results from (c) on a logarithmic scale. Cyan (orange) color corresponds to the numerical results of the real (imaginary) parts in (c) and (d). The slope is $1 / 3$ in (d). The system parameters are $\gamma=J=1$.

(a)

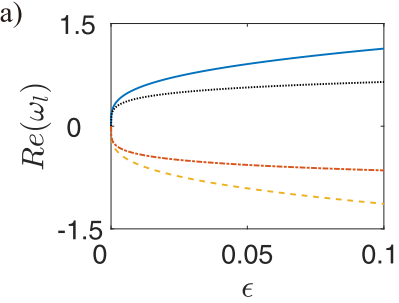

(b)

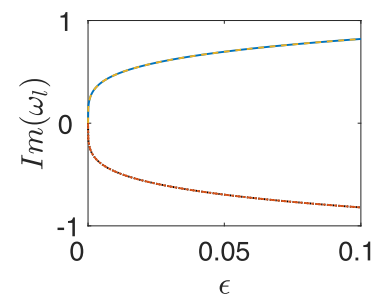

(c)

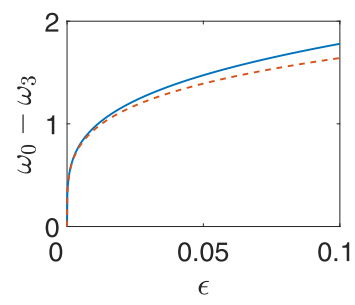

(d)

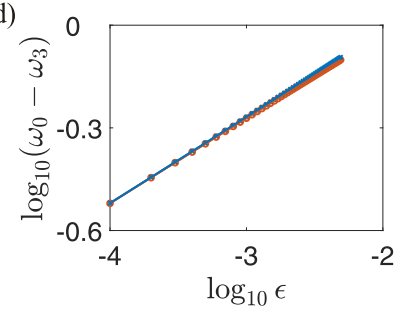

FIG. 6. Energy levels and frequency splittings near the EP4. (a) Real and (b) imaginary parts of four eigenenergies with different colors and line styles. (c) Frequency splitting between $\omega_{0}$ and $\omega_{3}$. (d) Results from (c) on a logarithmic scale. Cyan (orange) color corresponds to numerical results of the real (imaginary) parts of the SUSY array with $N=4$ in (c) and (d). The slope is $1 / 4$ in (d). The system parameters are $\gamma=J=1$.

\section{APPENDIX C: EIGENFREQUENCY RESPONSE TO PERTURBATION NEAR THE EP3, EP4, AND EP5}

Imposing perturbation $\epsilon$ on the couplings of the first two resonators, we can get the matrix form for Hamiltonians $H_{3 \times 3}$, $H_{4 \times 4}$ and $H_{5 \times 5}$ :

$$
H_{3 \times 3}=\left(\begin{array}{ccc}
\omega_{0}+2 i \gamma & \sqrt{2} J+\epsilon & 0 \\
\sqrt{2} J+\epsilon & \omega_{0} & \sqrt{2} J \\
0 & \sqrt{2} J & \omega_{0}-2 i \gamma
\end{array}\right),
$$

(a)

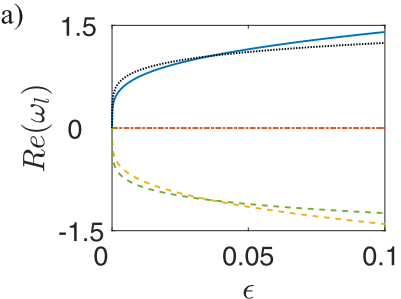

(c)

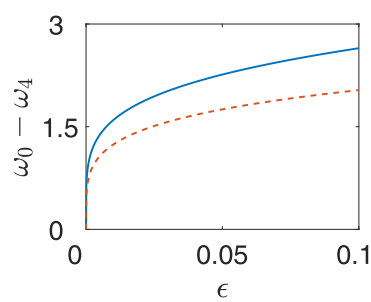

(b)

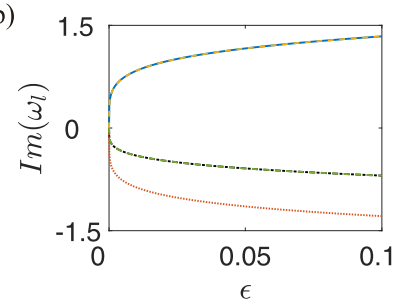

(d)

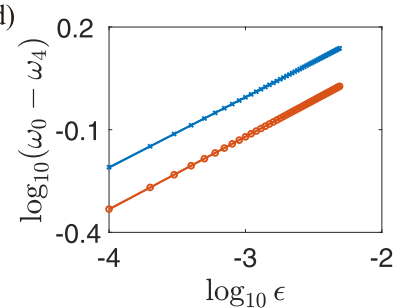

FIG. 7. Energy levels and frequency splittings near the EP5. (a) Real and (b) imaginary parts of five eigenenergies with different colors and line styles. (c) Frequency splitting between $\omega_{0}$ and $\omega_{4}$. (d) Results from (c) on a logarithmic scale. Cyan (orange) color corresponds to numerical results of the real (imaginary) parts in (c) and (d). The slope is $1 / 5$ in (d). The system parameters are $\gamma=J=1$. 


$$
H_{4 \times 4}=\left(\begin{array}{cccc}
\omega_{0}+3 i \gamma & \sqrt{3} J+\epsilon & 0 & 0 \\
\sqrt{3} J+\epsilon & \omega_{0}+i \gamma & 2 J & 0 \\
0 & 2 J & \omega_{0}-i \gamma & \sqrt{3} J \\
0 & 0 & \sqrt{3} J & \omega_{0}-3 i \gamma
\end{array}\right) \text {, }
$$

$$
\begin{aligned}
& H_{5 \times 5} \\
& =\left(\begin{array}{ccccc}
\omega_{0}+4 i \gamma & 2 J+\epsilon & 0 & 0 & 0 \\
2 J+\epsilon & \omega_{0}+2 i \gamma & \sqrt{6} J & 0 & 0 \\
0 & \sqrt{6} J & \omega_{0} & \sqrt{6} J & 0 \\
0 & 0 & \sqrt{6} J & \omega_{0}-2 i \gamma & 2 J \\
0 & 0 & 0 & 2 J & \omega_{0}-4 i \gamma
\end{array}\right) .
\end{aligned}
$$

Figure 5(c) represents the frequency splittings of bands with cyan solid and yellow dashed lines for $H_{3 \times 3}$, the real and imaginary parts of the energy levels are depicted in Figs. 5(a) and 5(b). The logarithmic relationship between the frequency splitting and disturbance is shown in Fig. 5(d), where the slope $1 / 3$ indicates the order of EP3.

Two energy bands with the maximum and minimum real parts in Fig. 6(a) have identical positive imaginary parts in Fig. 6(b); the middle two bands in Fig. 6(a) correspond to the same negative imaginary parts in Fig. 6(b). Figure 6(c) represents the frequency splittings of energy bands with cyan solid and orange dash-dot lines for $H_{4 \times 4}$, the real and imaginary parts of the energy levels are depicted in Figs. 6(a) and 6(b). The corresponding logarithmic relationship with $\epsilon$ is shown in Fig. 6(d), where the slope 1/4 indicates the order of EP4.

Figure 7(c) represents the frequency splittings of energy bands with cyan solid and green dashed lines for $H_{5 \times 5}$, the real and imaginary parts of the energy levels are depicted in Figs. 7(a) and 7(b). The logarithmic relationship between the frequency splitting and perturbation is shown in Fig. 7(d), where the slope $1 / 5$ indicates the order of EP5.
[1] C. M. Bender, Making sense of non-Hermitian Hamiltonians, Rep. Prog. Phys. 70, 947 (2007).

[2] N. Moiseyev, Non-Hermitian Quantum Mechanics (Cambridge University Press, Cambridge, UK, 2011).

[3] A. Krasnok, D. Baranov, H. Li, M.-A. Miri, F. Monticone, and A. Alú, Anomalies in light scattering, Adv. Opt. Photonics 11, 892 (2019).

[4] L. Feng, Y.-L. Xu, W. S. Fegadolli, M.-H. Lu, J. E. B. Oliveira, V. R. Almeida, Y.-F. Chen, and A. Scherer, Experimental demonstration of a unidirectional reflectionless paritytime metamaterial at optical frequencies, Nat. Mater. 12, 108 (2012).

[5] S. K. Gupta, Y. Zou, X.-Y. Zhu, M.-H. Lu, L.-J. Zhang, X.-P. Liu, and Y.-F. Chen, Parity-time symmetry in non-Hermitian complex optical media, Adv. Mater., 1903639 (2019).

[6] A. Guo, G. J. Salamo, D. Duchesne, R. Morandotti, M. VolatierRavat, V. Aimez, G. A. Siviloglou, and D. N. Christodoulides, Observation of $\mathcal{P} \mathcal{T}$-Symmetry Breaking in Complex Optical Potentials, Phys. Rev. Lett. 103, 093902 (2009).

[7] C. E. Rüter, K. G. Makris, R. El-Ganainy, D. N. Christodoulides, M. Segev, and D. Kip, Observation of parity-time symmetry in optics, Nat. Phys. 6, 192 (2010).

[8] B. Peng, Ş. K. Özdemir, F. Lei, F. Monifi, M. Gianfreda, G. L. Long, S. Fan, F. Nori, C. M. Bender, and L. Yang, Parity-timesymmetric whispering-gallery microcavities, Nat. Phys. 10, 394 (2014).

[9] L. Feng, Z. J. Wong, R.-M. Ma, Y. Wang, and X. Zhang, Singlemode laser by parity-time symmetry breaking, Science 346, 972 (2014).

[10] H. Hodaei, M.-A. Miri, M. Heinrich, D. N. Christodoulides, and M. Khajavikhan, Parity-time-symmetric microring lasers, Science 346, 975 (2014).

[11] L. Feng, R. El-Ganainy, and L. Ge, Non-Hermitian photonics based on parity-time symmetry, Nat. Photonics 11, 752 (2017).

[12] S. Longhi, Parity-time symmetry meets photonics: A new twist in non-Hermitian optics, Europhys. Lett. 120, 64001 (2017).
[13] R. El-Ganainy, K. G. Makris, M. Khajavikhan, Z. H. Musslimani, S. Rotter, and D. N. Christodoulides, NonHermitian physics and PT symmetry, Nat. Phys. 14, 11 (2018).

[14] M.-A. Miri and A. Alù, Exceptional points in optics and photonics, Science 363, eaar7709 (2019).

[15] Ş. K. Özdemir, S. Rotter, F. Nori, and L. Yang, Parity-time symmetry and exceptional points in photonics, Nat. Mater. 18, 783 (2019).

[16] Y. Wu, W. Liu, J. Geng, X. Song, X. Ye, C.-K. Duan, X. Rong, and J. Du, Observation of parity-time symmetry breaking in a single-spin system, Science 364, 878 (2019).

[17] K. G. Makris, R. El-Ganainy, D. N. Christodoulides, and Z. H. Musslimani, Beam Dynamics in $\mathcal{P} \mathcal{T}$ Symmetric Optical Lattices, Phys. Rev. Lett. 100, 103904 (2008).

[18] L. Xiao, X. Zhan, Z. H. Bian, K. K. Wang, X. Zhang, X. P. Wang, J. Li, K. Mochizuki, D. Kim, N. Kawakami, W. Yi, H. Obuse, B. C. Sanders, and P. Xue, Observation of topological edge states in parity-time-symmetric quantum walks, Nat. Phys. 13, 1117 (2017).

[19] L. Xiao, K. Wang, X. Zhan, Z. Bian, K. Kawabata, M. Ueda, W. Yi, and P. Xue, Observation of Critical Phenomena in ParityTime-Symmetric Quantum Dynamics, Phys. Rev. Lett. 123, 230401 (2019).

[20] K. Wang, X. Qiu, L. Xiao, X. Zhan, Z. Bian, W. Yi, and P. Xue, Simulating Dynamic Quantum Phase Transitions in Photonic Quantum Walks, Phys. Rev. Lett. 122, 020501 (2019); K. Wang, X. Qiu, L. Xiao, X. Zhan, Z. Bian, B. C. Sanders, W. Yi, and P. Xue, Observation of emergent momentum-time skyrmions in parity-time-symmetric non-unitary quench dynamics, Nat. Commun. 10, 2293 (2019).

[21] S. Klaiman, U. Günther, and N. Moiseyev, Visualization of Branch Points in $\mathcal{P} \mathcal{T}$-Symmetric Waveguides, Phys. Rev. Lett. 101, 080402 (2008).

[22] J. Doppler, A. A. Mailybaev, J. Böhm, U. Kuhl, A. Girschik, F. Libisch, T. J. Milburn, P. Rabl, N. Moiseyev, and S. Rotter, 
Dynamically encircling an exceptional point for asymmetric mode switching, Nature (London) 537, 76 (2016).

[23] H. Xu, D. Mason, L. Jiang, and J. G. E. Harris, Topological energy transfer in an optomechanical system with exceptional points, Nature (London) 537, 80 (2016).

[24] S. Assawaworrarit, X. Yu, and S. Fan, Robust wireless power transfer using a nonlinear parity-time-symmetric circuit, Nature (London) 546, 387 (2017).

[25] B. Midya, H. Zhao, and L. Feng, Non-Hermitian photonics promises exceptional topology of light, Nat. Commun. 9, 2674 (2018).

[26] S. Cao and Z. Hou, Angular-Asymmetric Transmitting Metasurface and Splitter for Acoustic Waves: Combining the Coherent Perfect Absorber and a Laser, Phys. Rev. Appl. 12, 064016 (2019).

[27] P. Miao, Z. Zhang, J. Sun, W. Walasik, S. Longhi, N. M. Litchinitser, and L. Feng, Orbital angular momentum microlaser, Science 353, 464 (2016).

[28] S. Longhi and L. Feng, Unidirectional lasing in semiconductor microring lasers at an exceptional point, Photonics Res. 5, B1 (2017).

[29] W. Chen, Ş. K. Özdemir, G. Zhao, J. Wiersig, and L. Yang, Exceptional points enhance sensing in an optical microcavity, Nature (London) 548, 192 (2017).

[30] H. Hodaei, A. U. Hassan, S. Wittek, H. Garcia-Gracia, R. El-Ganainy, D. N. Christodoulides, and M. Khajavikhan, Enhanced sensitivity at higher-order exceptional points, Nature (London) 548, 187 (2017).

[31] B. Peng, Ş. K. Özdemir, M. Liertzer, W. Chen, J. Kramer, H. Yilmaz, J. Wiersig, S. Rotter, and L. Yang, Chiral modes and directional lasing at exceptional points, Proc. Natl. Acad. Sci. U.S.A. 113, 6845 (2016).

[32] L. Jin and Z. Song, Incident Direction Independent Wave Propagation and Unidirectional Lasing, Phys. Rev. Lett. 121, 073901 (2018).

[33] J. Wiersig, Enhancing the Sensitivity of Frequency and Energy Splitting Detection by Using Exceptional Points: Application to Microcavity Sensors for Single-Particle Detection, Phys. Rev. Lett. 112, 203901 (2014).

[34] M. Am-Shallem, R. Kosloff, and N. Moiseyev, Parameter estimation in atomic spectroscopy using exceptional points, Phys. Rev. A 93, 032116 (2016).

[35] Z.-P. Liu, J. Zhang, Ş. K. Özdemir, B. Peng, H. Jing, X.Y. Lü, C.-W. Li, L. Yang, F. Nori, and Y. X. Liu, Metrology with $\mathcal{P} \mathcal{T}$-Symmetric Cavities: Enhanced Sensitivity Near the $\mathcal{P} \mathcal{T}$-Phase Transition, Phys. Rev. Lett. 117, 110802 (2016).

[36] H.-K. Lau and A. A. Clerk, Fundamental limits and nonreciprocal approaches in non-Hermitian quantum sensing, Nat. Commun. 9, 4320 (2018).

[37] M. Zhang, W. Sweeney, C. W. Hsu, L. Yang, A. D. Stone, and L. Jiang, Quantum Noise Theory of Exceptional Point Amplifying Sensors, Phys. Rev. Lett. 123, 180501 (2019).

[38] P. Djorwe, Y. Pennec, and B. Djafari-Rouhani, Exceptional Point Enhances Sensitivity of Optomechanical Mass Sensors, Phys. Rev. Appl. 12, 024002 (2019).

[39] Y.-H. Lai, Y.-K. Lu, M.-G. Suh, Z. Yuan, and K. Vahala, Observation of the exceptional-point-enhanced Sagnac effect, Nature (London) 576, 65 (2019).
[40] M. P. Hokmabadi, A. Schumer, D. N. Christodoulides, and M. Khajavikhan, Non-Hermitian ring laser gyroscopes with enhanced Sagnac sensitivity, Nature (London) 576, 70 (2019).

[41] Y. Cao and P. Yan, Exceptional magnetic sensitivity of $\mathcal{P} \mathcal{T}$ symmetric cavity magnon polaritons, Phys. Rev. B 99, 214415 (2019).

[42] G.-Q. Zhang, Y.-P. Wang, and J. Q. You, Dispersive readout of a weakly coupled qubit via the parity-time-symmetric phase transition, Phys. Rev. A 99, 052341 (2019).

[43] C. Chen, L. Jin, and R.-B. Liu, Sensitivity of Parameter Estimation near the Exceptional point of a non-Hermitian system, New J. Phys. 21, 083002 (2019).

[44] J. Schnabel, H. Cartarius, J. Main, G. Wunner, and W. D. Heiss, Simple models of three coupled $\mathcal{P} \mathcal{T}$-symmetric wave guides allowing for third-order exceptional points, Acta Polytech. 57, 454 (2017).

[45] G. Demange and E.-M. Graefe, Signatures of three coalescing eigenfunctions, J. Phys. A: Math. Theor. 45, 025303 (2012).

[46] G.-Q. Zhang and J. Q. You, Higher-order exceptional point in a cavity magnonics system, Phys. Rev. B 99, 054404 (2019).

[47] H. Jing, Ş. K. Özdemir, H. Lü, and F. Nori, High-order exceptional points in optomechanics, Sci. Rep. 7, 3386 (2017).

[48] K. Ding, G. Ma, M. Xiao, Z. Q. Zhang, and C. T. Chan, Emergence, Coalescence, and Topological Properties of Multiple Exceptional Points and Their Experimental Realization, Phys. Rev. X 6, 021007 (2016).

[49] L. Jin, Parity-time-symmetric coupled asymmetric dimers, Phys. Rev. A 97, 012121 (2018).

[50] S. Wang, B. Hou, W. Lu, Y. Chen, Z. Q. Zhang, and C. T. Chan, Arbitrary order exceptional point induced by photonic spin-orbit interaction in coupled resonators, Nat. Commun. 10, 832 (2019).

[51] Z. Bian, L. Xiao, K. Wang, X. Zhan, F. A. Onanga, F. Ruzicka, W. Yi, Y. N. Joglekar, and P. Xue, Time invariants across a fourth-order exceptional point in a parity-time-symmetric qudit, arXiv:1903.09806.

[52] X. Z. Zhang, L. Jin, and Z. Song, Perfect state transfer in $\mathcal{P} \mathcal{T}$ symmetric non-Hermitian networks, Phys. Rev. A 85, 012106 (2012).

[53] M. H. Teimourpour, R. El-Ganainy, A. Eisfeld, A. Szameit, and D. N. Christodoulides, Light transport in $\mathcal{P} \mathcal{T}$-invariant photonic structures with hidden symmetries, Phys. Rev. A 90, 053817 (2014).

[54] Y.-X. Xiao, Z.-Q. Zhang, Z. H. Hang, and C. T. Chan, Anisotropic exceptional points of arbitrary order, Phys. Rev. B 99, 241403(R) (2019).

[55] L. Pan, S. Chen, and X. Cui, High-order exceptional points in ultracold Bose gases, Phys. Rev. A 99, 011601(R) (2019)

[56] E. M. Graefe, U. Günther, H. J. Korsch, and A. E. Niederle, A non-Hermitian $\mathcal{P} \mathcal{T}$ symmetric Bose-Hubbard model: Eigenvalue rings from unfolding higher-order exceptional points, J. Phys. A: Math. Theor. 41, 255206 (2008).

[57] Q. Zhong, D. N. Christodoulides, M. Khajavikhan, K. G. Makris, and R. El-Ganainy, Power-law scaling of extreme dynamics near higher-order exceptional points, Phys. Rev. A 97, 020105(R) (2018).

[58] S. Longhi, Bloch oscillations in tight-binding lattices with defects, Phys. Rev. B 81, 195118 (2010). 
[59] L. Jin and Z. Song, A physical interpretation for the nonHermitian Hamiltonian, J. Phys. A: Math. Theor. 44, 375304 (2011).

[60] R. El-Ganainy, L. Ge, M. Khajavikhan, and D. N. Christodoulides, Supersymmetric laser arrays, Phys. Rev. A 92, 033818 (2015).

[61] M. P. Hokmabadi, N. S. Nye, R. El-Ganainy, D. N. Christodoulides, and M. Khajavikhan, Supersymmetric laser arrays, Science 363, 623 (2019).

[62] B. Midya, H. Zhao, X. Qiao, P. Miao, W. Walasik, Z. Zhang, N. M. Litchinitser, and L. Feng, Supersymmetric microring laser arrays, Photonics Res. 7, 363 (2019).

[63] M. Heinrich, M.-A. Miri, S. Stuitzer, R. El-Ganainy, S. Nolte, A. Szameit, and D. N. Christodoulides, Supersymmetric mode converters, Nat. Commun. 5, 3698 (2014).

[64] S. Weinberg, The Quantum Theory of Fields, Vol. 3: Supersymmetry (Cambridge University Press, Cambridge, UK, 2000).

[65] M. Christandl, N. Datta, A. Ekert, and A. J. Landahl, Perfect State Transfer in Quantum Spin Networks, Phys. Rev. Lett. 92, 187902 (2004).
[66] A. Pick, S. Silberstein, N. Moiseyev, and N. Bar-Gill, Robust mode conversion in NV centers using exceptional points, Phys. Rev. Res. 1, 013015 (2019).

[67] Z. Xiao, H. Li, T. Kottos, and A. Alù, Enhanced Sensing and Nondegraded Thermal Noise Performance Based on $\mathcal{P} \mathcal{T}$ Symmetric Electronic Circuits with a Sixth-Order Exceptional Point, Phys. Rev. Lett. 123, 213901 (2019).

[68] M. Müller and I. Rotter, Exceptional points in open quantum systems, J. Phys. A: Math. Theor. 41, 244018 (2008).

[69] H. Eleuch and I. Rotter, Clustering of exceptional points and dynamical phase transitions, Phys. Rev. A 93, 042116 (2016).

[70] B.-B. Wei and L. Jin, Universal critical behaviours in non-Hermitian phase transitions, Sci. Rep. 7, 7165 (2017).

[71] Y. Ma and A. Edelman, Nongeneric Eigenvalue perturbations of Jordan blocks, Linear Algebra Appl. 273, 45 (1998).

[72] T. Kato, Perturbation Theory for Linear Operators (Springer, Berlin, 1966). 